\title{
Nurse adaptability: Implementing clinical trials in the midst of a pandemic
}

\author{
Sarah J. Mendez, Brian Raimondo, Patricia Hughes
}

NYU Langone Health, New York, NY, USA

Received: September 14, 2020

Accepted: December 1, 2020

Online Published: December 16, 2020

DOI: $10.5430 /$ jnep.v11n $4 \mathrm{p} 30$

URL: https://doi.org/10.5430/jnep.v11n4p30

\begin{abstract}
Working in Manhattan, the center of the nations' outbreak of the novel coronavirus-19 virus truly demonstrated how adaptable nurses are. During this time, multiple clinical research trials began at our academic medical center, NYU Langone Health, as researchers attempted to learn what medical interventions worked best to treat critically-ill COVID-19 patients. In designing and implementing these trials, the researchers had little familiarity with the workings of inpatient hospital units. They did not understand how nursing staff provided care to patients on these units. Likewise, many bedside nurses had never assisted researchers in conducting clinical research on their patients. Therefore, a nursing operations team (NOT) was needed to assist both the research teams and the inpatient nurses. NOT met with the researchers to review proposed clinical research trials and determine how nursing staff would be utilized to complete the required research tasks such as specimen and data collection, study intervention administration, and patient monitoring. Toward that end, NOT developed education and training materials on all of the research trials that were implemented at NYU Langone Health for our bedside nurses. This education included tip sheets, safety huddle rounds with the involved units, and "just in time" education to any nurse whose patient was urgently enrolled in a trial. In this way, NOT helped bedside nurses quickly adapt to their role in assisting the research team conduct their studies on our COVID positive inpatients.
\end{abstract}

Key Words: Coronavirus, Covid-19, Nursing, Pandemic, Research trials

\section{INTRODUCTION}

Novel coronavirus-19 began to infect Americans in large numbers in early 2020. As it is a new virus there are no known treatments. Medical doctors and researchers immediately went into action developing clinical trials in an attempt to hinder the virus, using data from patients in China, which was ahead of the rest of the world in terms of familiarity with the virus.

Based on early clinical data, the virus seemed to trigger a significant cytokine release in infected individuals, causing a variety of inflammatory symptoms. ${ }^{[1]}$ Providers started look- ing at a variety of medications to lessen or possibly prevent these symptoms. These medications included IL-6 receptor antagonists, complement C5 inhibitors, anti-malarials, and a neutralizing IgG1 monoclonal antibody. ${ }^{[2]}$ Convalescent plasma, plasma obtained from individuals who were diagnosed with Covid-19 and recovered, was likewise under investigation to determine if the antibodies in the recovered patient provided a shorter and less severe duration of the virus to newly-diagnosed individuals. These potential treatment regimens were all in the planning stages for scientific research at various academic medical centers in the affected areas in the United States, including NYU Langone Health.

\footnotetext{
*Correspondence: Sarah J. Mendez; Email: Sarah.mendez@nyulangone.org; Address: NYU Perlmutter Cancer Center, 160 E. 34th St, 11th Floor, NY, NY 10016, USA.
} 


\section{BACKGROUND}

The NYU Langone Health Scientific Research Committee (SRC), comprised of medical doctors, medical researchers, data managers, research nurses, and program managers, was established at the onset of the Covid-19 clinical research trials at NYU. The SRC had a sub-group known as the Covid Clinical Research Planning Committee (CCRPC), which met daily to discuss feasibility of trials being approved by the institutional review board (IRB). The CCRPC's goal was to operationalize each trial in order to study the treatment of patients infected with Covid-19. The CCRPC developed a plan to determine where to roll out the various studies; however, simultaneously, the acuity level of the inpatient units was constantly changing affecting research decisions. Similar to other institutions in NYC, units that normally would not have been used for intensive care were converted to ICU beds. ${ }^{[3]}$ The CCRPC understood the need to implement these trials on inpatient units, but did not have a plan to communicate and educate the bedside nurses who would be assisting with these research trials for enrolled patients. Each research team consisted of the principal investigator (PI) or sub-PI, data manager, program manager or director, and a research nurse. Many medical students were recruited to assist with obtaining consent from the patients. Some members of each research team were working remotely which further confounded the communications with the nursing staff on a particular unit.

The CCRPC chair understood the need for real time education in order to implement Covid-19 research at the bedside. The Chief Nursing Officer tasked the Director of Nursing Professional Practice with organizing a nursing operations team (NOT) that would work in conjunction with the scientific research to ensure bedside nurses were prepared to administer research drugs/products. Three nurses were selected to become members of NOT and these were a Nursing Professional Development Specialist, an Oncology Clinical Nurse Specialist, and a Nursing Quality Specialist/Nursing Standards Coordinator. The reason for the creation of NOT was to ensure the staff nurses received all vital information in a timely manner and that they received educational and operational support when questions arose regarding the trials. NOT participated in daily phone meetings with the researchers, discussing each trial, patient enrollment, and any obstacles, real or perceived, in rolling out these studies to the various nursing units.

The bedside nurses caring for Covid patients consisted of acute care medical/surgical nurses and critical care nurses, neither of whom had experience in clinical research or the administration of an IL-6 receptor antagonist or management of their potential side effects. These medications fall under the category of immunotherapy which are only administered by chemotherapy competent nurses at our institution. In order to assist in operationalization of these trials, Nursing Leadership decided to allow all staff nurses to administer these research medications after they received education. These decisions were made in light of the fact that the medications were not deemed hazardous by the National Institute for Occupational Safety and Health (NIOSH) ${ }^{[4]}$ were FDA approved, and were being studied for an off-label use. See Figure 1 for a visual of the clinical trial implementation process.

\section{COMMUNiCATION WITH THE TEAMS}

Daily meetings with the CCRPC ensured a systematic process for research operationalization. When NOT was first established there were 3 studies that were being initiated and required education for nurses caring for the patient. These 3 trials consisted of two IL- 6 receptor antagonists and one antiviral, each to be administered by the bedside nurse.

As multiple trials were occurring within the hospital, the CCRPC wanted to assign trials to specific units. In addition, some trials were designed for acute patients, and some for ICU patients (e.g., ventilated patients). Therefore, the CCRPC developed a grid of the clinical units so that research teams were aware of the acuity level of each unit, i.e. acute vs critical care, in order to help in assigning trials. Due to changing circumstances in unit operations and patient acuity, this grid kept changing, which caused confusion with CCRPC as to where to roll out new/upcoming trials. Units that were initially designated acute care quickly turned into critical care units as patients began to deteriorate. Some specialty units, such as oncology, were moved to an entirely different building to allow for the opening of an additional Covid unit.

During these daily phone calls NOT emphasized that even though a trial was assigned to a specific unit, the patient may not remain on that unit for the duration of the trial, since patient transfers are a common occurrence (e.g., patient transfers from acute to intensive care as their clinical condition deteriorated). Unfortunately, this concept was not fully appreciated by the CCRPC, as researchers they were unaware of the intricate operations of a nursing unit.

\section{HOW THE TIP SHEETS WERE DEVISED}

In order to operationalize these trials, each study required a one-page summary of pertinent details that nurses needed to know prior to the start of the research trial. These tip sheets were derived from lengthy, detailed research study protocols that outlined each study. NOT was responsible for creating these tip sheets with the research team who reviewed them 
to ensure all study requirements were communicated. These tip sheets provided an overview of the medication or blood product being given, study specific specimen collection timelines, study specific requirements, and contact information for the study team for questions. Each research study protocol ranged from 25 to 50 pages of detail, most of which was not relevant to the staff nurses caring for these patients.

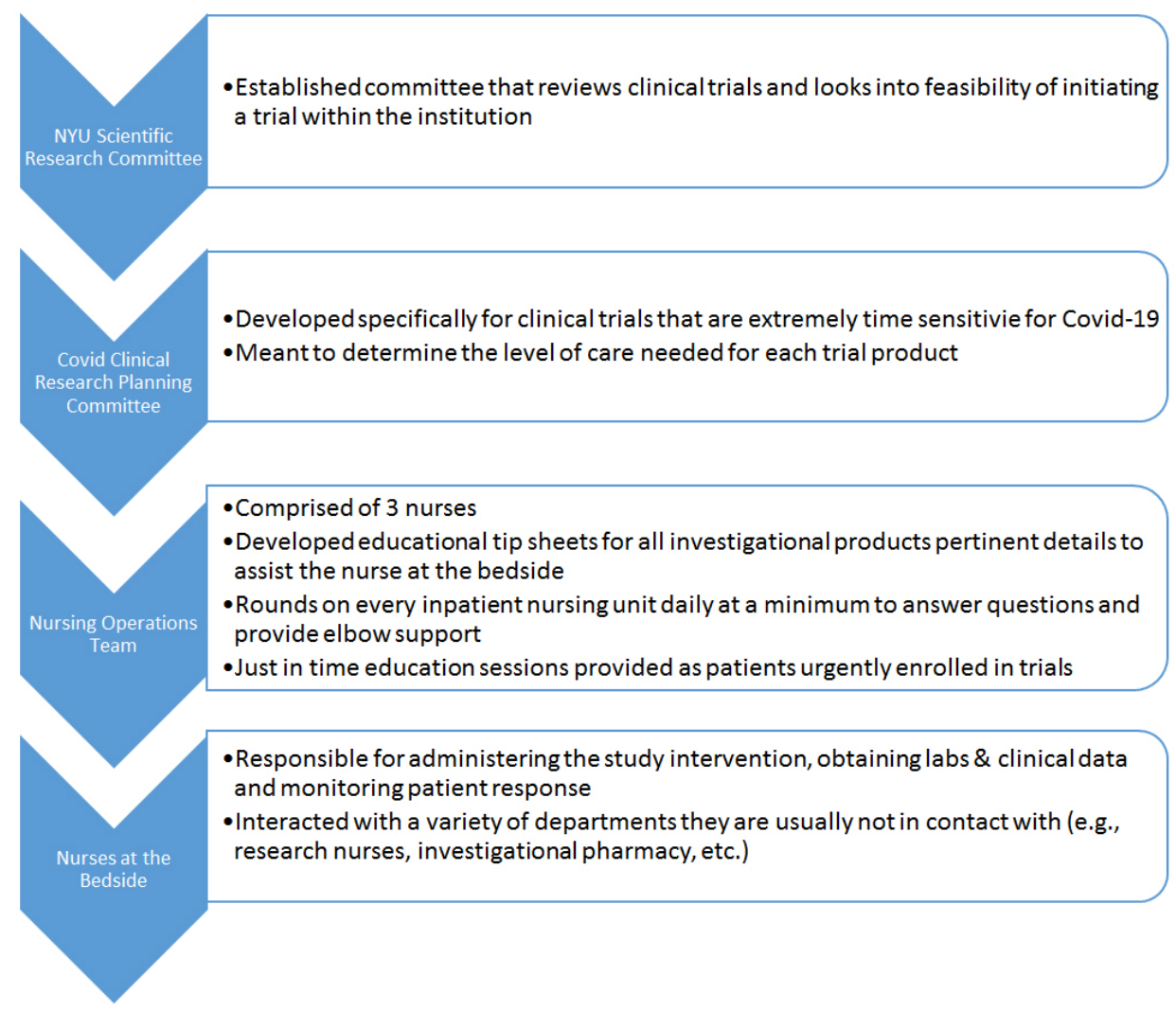

Figure 1. Flow of research trial education

Editing these protocols down to a single page was challenging but imperative, since the frontline nurses were already overwhelmed with patient care responsibilities. PIs and their teams who felt strongly that the nurses needed to know pharmacotherapeutics of the drug developed short videos made available via our online e-learning system for nurses to watch at their convenience.

The NOT-created tip sheets were individualized for each trial and then provided to the nurses in a variety of ways: they were given individually-printed copies as patients became enrolled in trials, laminated copies were available on the assigned unit, and finally were placed on the hospital intranet site. Due to the continued evolving nature of the units' acuity level, NOT decided that all tip sheets were to be distributed to every unit in the institution as a patient on a specific trial may end up on a different unit. Additionally, the tip sheets were presented at safety huddles on the units and/or, if needed, there would be "just-in-time" education at the bedside.

While working on these tip sheets there was much discus- sion between NOT and the research team to ensure correct information was provided, and to determine the best ways to collect pertinent laboratory specimens or ensure required patient monitoring was performed, which minimized nursing workflow interruption. For example, laboratory specimen collection could have presented a challenge to nurses who were experiencing increased patient assignments, but through nursing and research collaboration, consensus was obtained. In addition, NOT and the research team made every effort to ensure specimen collection times would not require frequent entry into patient rooms, to allow for PPE preservation and minimal nurse exposure. Researchers were agreeable to modifying time of collection and patient monitoring whenever possible, to coincide with administration of the trial medication or with morning blood work.

\section{Challenges encountered}

Specimen collection and patient monitoring presented some initial challenges, but others emerged as various trials were operationalized. These challenges included, but were not lim- 
ited to, maintaining study blindness, nursing documentation, and adjustments to protocols mid-trial.

\subsection{Convalescent plasma research trial}

Convalescent plasma proved to be the most challenging trial in regards to implementation. This research team was extremely proactive in initiating the study, as time was of the essence to study this intervention; unfortunately they did not anticipate potential issues that accompany blood product transfusions in general. When this blinded study was first presented, the placebo was to be a unit of fresh frozen plasma. However, when it came to study implementation, it was decided that the use of a unit of plasma was not the optimal choice. It was decided that the placebo would be a $250 \mathrm{~mL}$ bag of Normal Saline (NS). With this significant change in trial design, more challenges presented themselves. These included maintaining blindness of the study, and how to document the convalescent plasma versus the NS placebo. As to blindness, plasma generally is a yellowish straw colored product while NS is a perfectly clear solution. It would be apparent to either the patient or the research team what was being transfused. The second complication for this study was the documentation in the electronic medical record. Blood product administration is documented in one area of the patient record; whereas, normal saline is documented as a medication. NOT and the researchers worked together to brainstorm solutions to these issues.

The difficulties with documentation occurred as our institution uses an electronic blood administration record (BAR). Every blood product dispensed from Blood Bank arrives to the unit with a paper BAR attached, which is only used during electronic downtime. All blood products undergo a two clinician verification and are scanned into the electronic medical record. Normal saline is treated as a medication and is documented in the medication administration record (MAR). Therefore, anyone who had access to the patient chart would immediately know if the patient received the actual convalescent plasma or the placebo, thereby unblinding the study. To resolve this issue, NOT met with the convalescent plasma research team and Blood Bank to determine best approach to avoid this potential problem. It was decided that the plasma would be documented via the standard route (e.g., electronic scanning into the electronic health record); and the NS would utilize the paper BAR. The RNs would also use their clinical mobile device to take a clinical picture of the paper BAR, which was then uploaded into the electronic medical record. Further documentation was implemented where the nurse entered a nursing progress note using a smart phrase. Use of the smart phrase allowed the $\mathrm{RN}$ to create a standardized note, which documented when the product was administered

Published by Sciedu Press and whether the patient had a reaction. Nurses were also instructed to ensure start and stop times of the transfusion were entered into the intake/output flowsheet.

To address the issue of color differences between the convalescent plasma versus NS placebo Blood Bank purchased amber tubing covers. The nurse needed amber colored tubing to cover the IV line from top to bottom and used an opaque bag (already distributed by Blood Bank with all blood products) to cover the product whether it be plasma or NS. NOT worked with the Blood Bank to work out how the amber tubing would be cut and distributed and also with nursing staff to communicate the proper way it would be used. The Blood Bank special ordered these amber tubing covers to be sent to the unit with the research product. Once the Blood Bank received the covers it was determined that two separate pieces needed to be sent to ensure coverage of line above and below the infusion pump.

One unforeseen event during the trial occurred when the nurse caring for the research patient contacted the research team to ask a question about convalescent plasma, which unblinded this one subject. Secondary to that occurrence it was determined that the primary team was to be contacted if there was an adverse reaction to, or questions about, the plasma.

Blood samples for this study also posed a complication as the collection process was different from standard practice. The collected specimens, that were specific to this trial and not standard of care, were to be sent to a separate research lab where the trial originated and where the PI is located. NOT, the research team, and the laboratory manager had multiple conference calls to determine the best way to obtain and process these samples. A previously used method of printing laboratory requisitions for specific labs that were labeled miscellaneous had been phased out. However, due to the complexity of finding an alternative way to identify these specific specimens it was determined that the previous printing method for lab requisition was to be used. The tip sheet was adjusted, the nurses were made aware, and the lab technicians were notified of the change in practice for these specific specimens.

\subsection{Hydroxychloroquine research trial}

Some research trials incorporated oral medications, including the use of hydroxychloroquine (HCQ) which is used in the treatment of malaria. A majority of the patients admitted through the Emergency Department were prescribed HCQ the moment they tested positive for Covid-19. Unfortunately, this was a hindrance to enrolling subjects for this trial as they needed to be naïve to this medication in order to be enrolled. 
The providers needed to communicate with their counterparts in the Emergency Department to make them aware of this study, in an attempt to have more patients eligible for the new HCQ trial.

Due to the rapid nature of implementation for many of these research trials there was not always adequate time to obtain a perfect placebo. The placebo in this trial was not a perfect match to the trial medication. Education was provided by the sub-PI regarding the differences in appearance and since staff were familiar with the look of HCQ they were asked not to disclose to the patient what was being administered, even if obvious to them.

\subsection{Remdesivir research trial}

Remdesivir, an antiviral that has been in use for a number of years, was one of the first trials initiated at our institution. This trial demonstrated that no matter how simple a trial design may seem on paper, it often never is so. One requirement for the trial protocol was the collection of a nasopharyngeal/oropharyngeal swab prior to each dose of the trial drug or placebo. One of the original units assigned to this trial raised the issue of storing nasopharyngeal/oropharyngeal swabs post collection. The initial concern was how and where to store the swab prior to getting it to the lab. This issue arose when we were in the early stages of the pandemic and there was so much still unknown about how the virus was transmitted. Through discussions with the staff and members of the research team it was determined that the individual delivering the medication/placebo would remain on the unit while the nurse obtained the specimen: they would then walk the specimen to the lab. This solution not only prevented the swab from being stored on the unit for any length of time, it also enabled the nurse to collect the specimen while initiating the infusion thus removing the need for an additional trip into the patients' room. As with most of these trials, the goal was to reduce any extra trips into a patient room and to maintain standard of care whenever possible.

\subsection{Remdesivir II research trial}

The second phase of the original remdesivir trial included the use of baricitinib, an oral medication used for rheumatoid arthritis. While the issue of the nasopharyngeal/oropharyngeal swab collection and transport to the lab had been resolved, a new issue arose with regards to the administration of baricitinib. Baricitinib while in intact oral form is not considered a hazardous medication, however once it is broken/crushed, it is considered a hazardous medication. For the patients who were on this trial and became intubated or were unable to swallow whole pills for any reason, a "slurry" had to be made. NOT arranged a phone call with the investigational pharmacy and the inpatient pharmacist on the transplant service, to discuss best options for ensuring the patient received the trial medication in an appropriate manner based on their physical status. For safety and regulatory reasons, the nurses on the units are not allowed to crush hazardous medications. This can only be done by pharmacy, where there are containment hoods for this purpose. The investigational pharmacy was responsible for compounding the pill into the slurry, if the patient were to receive the medication via gastrostomy tube or could not ingest a whole pill. This phase of the study looked to enroll 15 subjects, which made managing this unexpected challenge reasonable. The inpatient pharmacist was agreeable to assisting this process through an email to the investigational pharmacy; the inpatient pharmacist would review the enrolled patients on a daily basis and email the investigational pharmacy regarding any patient who was unable to swallow whole pills. If a nurse was caring for a patient who required the baricitinib to be made into a slurry, they were educated on how to administer it. It was explained that the medication was to be given directly from the syringe it was delivered in and not mixed into any food or drink to ensure the full dose was provided to patient.

\section{OUTCOMES FOR NURSING}

When the pandemic first started, there was uncertainty in how to care for COVID patients. Nurses often felt helpless and were chasing symptoms and managing them to the best extent possible, trying to prevent clinical deterioration and death. Although one was only able to see the eyes of any individual due to their extensive PPE, there was a look of despair in staff due to the inability of getting ahead of the virus. These research trials provided a sense of hope to the frontline nurses who were seeing mortality on a scale not witnessed in their lifetimes.

The ability of nurses to adapt to change without warning was demonstrated throughout this process. Seeing firsthand how the nursing staff was able to adapt to all of the trials with barely any interruptions in caring for their patients was astonishing. This pandemic reinforced the resiliency of our nursing staff in times of stress. This resiliency was evidenced by the nurses being able to make instantaneous variations to their normal routine to ensure medications were given properly, and that lab specimens were sent to the correct department.

When NOT rounds on the inpatient units, the nurses did not flee from sight as per usual; it was never a "what do they want now" discussion, rather a discussion regarding whether we had any results to share from the various studies or if there were new trials starting with different medications. Of- 
tentimes, nurses on one unit would share stories of patients being discharged from other units. They had hope that their patients would recover. They felt strongly that enrollment of their patients in these research trials would help them make it to a place of health. This was further proof of how amazing the frontline nurses were and are to this day.

The interdisciplinary collaboration and respect was a winwin for all involved: researchers, doctors, pharmacists, and nurses all working toward the same goal and their willingness to discuss best options for caring for these patients was beneficial. This was a revelation for the doctors and researchers, some of whom had never been on an inpatient unit before, as to the inner workings of a nursing unit. While each unit has its own little intricacies the overall functioning of clinical units remains the same regardless of location.

Going forward from this invaluable experience, there is now a plan in place in the event the hospital needs to operationalize research trials rapidly regardless of the situation requiring it. The various departments involved in these trials now have contacts in each operational group. The collaboration also crossed our health system campuses between NYC, Brooklyn, and Long Island with key players identified at each one. While this type of plan is necessary, the reality of any situation requiring it is not something any of us would anticipate happening again.

We believe that this was an invaluable experience. Being able to share this experience with others will clearly demonstrate how effectively people of varying degrees and backgrounds can work together during difficult times. We proved that we are, as an institution, NY Strong and capable of making the impossible look, if not simple, then manageable.

\section{ACKNOWLEDGEMENTS}

This article is dedicated to all the frontline nurses at NYU Langone Health whose unwavering dedication and professionalism toward their patients contributed to a better understanding of a deadly virus. These research trials would not have been possible without them.

\section{CONFLICTS OF INTEREST DISCLOSURE}

The authors declare that there is no conflict of interest.

\section{REFERENCES}

[1] Kox M, Waalders NJB, Kooistra EJ, et al. Cytokine levels in critically ill patients with COVID-19 and other conditions. Journal of American Medical Association. 2020 September; E1-E2.

[2] Gandhi RT, Lynch JB, del Rio C. Mild or moderate covid-19. New England Journal of Medicine. 2020 April; 1-9.
[3] Peters AW, Chawla KS, Turnbull ZA. Transforming ORs into ICUs. New England Journal of Medicine. 2020 May; 382: e52(1)-e52(2). PMid:32329973 https : //doi.org/10.1056/NEJMc2010853

[4] NIOSH List of Antineoplastic and Other Hazardous Drugs in Healthcare Settings. 2016. Available from: https ://www.cdc.gov/nios h/docs/2016-161/default.html 